\title{
Simulating the effects of wolf-elk population dynamics on resource flow to scavengers
}

\author{
Christopher C. Wilmers*, Wayne M. Getz \\ Department of Environmental Science, Policy and Management, 201 Wellman Hall \#3112, \\ University of California, Berkeley, CA 94720-3112, USA
}

Received 2 September 2003; received in revised form 30 January 2004; accepted 16 February 2004

\begin{abstract}
The reintroduction of gray wolves to Yellowstone National Park (YNP) provides a natural experiment regarding the effects of top predators on scavenger species. Fieldwork on the Northern Range of Yellowstone indicates that wolves facilitate carrion acquisition by scavengers, but it is unclear whether this represents a transient or permanent effect of wolf reintroduction. Here we present a wolf-elk model with human elk harvest and use it to investigate the long-term consequences of predator-prey dynamics and hunting on resource flow to scavengers. Our model shows that while wolves reduce the total amount of carrion, they stabilize carrion abundance by reducing temporal variation in the quantity of carrion and extending the period over which carrion is available. Specifically, the availability of carrion is shifted from reliance on winter severity and elk density to dependence on the strength of wolf predation. Though wolves reduce the overall abundance of carrion by lowering the elk population, this reduction is partially offset by increases in the productivity of an elk population invigorated by removal of the weakest individuals. The result of this is higher carrion production per elk in the presence of wolves. In addition, this yields an ecological explanation for the phenomena that predators increase the robustness of their prey: namely that by reducing the effect of density-dependent resource competition among elk, those that remain, even some of the older animals, are better fed and healthier as a result. Our model also suggests that human hunting has no effect on the distribution of carrion across the year but is crucial in determining the long-term abundance of carrion because of the effect of hunting on elk population levels. By reducing the proportion of cows in the annual hunt, which have historically been high in order to control the number of elk migrating north of the park, managers can allow an adequate supply of carrion without substantially reducing hunter take. The effects of a more tractable food resource is likely to benefit scavengers in Yellowstone and other areas of the world where wolves have been or are currently being considered for reintroduction.
\end{abstract}

(C) 2004 Elsevier B.V. All rights reserved.

Keywords: Subsidy; Population dynamics; Scavenging; Canis lupus; Matrix model; Density dependence; Yellowstone; Hunting

\section{Introduction}

The management and conservation of top predators and their respective prey is often informed by com-

\footnotetext{
* Corresponding author. Tel.: +1-510-393-0290; fax: +1-510-642-7428.

E-mail address: cwilmers@nature.berkeley.edu (C.C. Wilmers).
}

munity theory on predator feeding habits. By curbing the number or altering the behavior of prey, predators have been shown to cause the release of species two rungs down the food chain (Estes and Palmisano, 1974; Power, 1990; McLaren and Peterson, 1994; Ripple et al., 2001). In addition, predators may also facilitate the coexistence of other prey species by suppressing competitive dominants (Paine, 1966; Inouye et al., 1980; McNaughton, 1983). While the positive 
indirect effects of predators eating prey on basal levels of the food chain have been well documented both theoretically and empirically, little work has been undertaken regarding the indirect effects of top predator feeding habits on other guild members. Recent studies on large carnivores have revealed that predators may also help to shape scavenger communities by mediating their supply of carrion (Carbone et al., 1997; Wilmers et al., 2003b). Understanding these effects is crucial to the proper management of ecosystems.

Reintroduced gray wolves (Canis lupus) in Yellowstone National Park (YNP) have been shown to provide a temporal subsidy to scavengers by transferring the availability of carrion from the resource-rich end of winter period to the resource-poor early winter period (Wilmers et al., 2003a). Scavengers in Yellowstone historically experienced a boom in food supply at the end of severe winters when elk (Cervus elaphus) weakened and died, but received very little carrion the rest of the year or in mild winters (Houston, 1978; Gese et al., 1996). With the reintroduction of gray wolves to Yellowstone in 1995 (Bangs and Fritts, 1996), scavenge of partially consumed wolf-killed carcasses became more constant through time and more abundant overall (Wilmers et al., 2003a). By reducing the peaks in carrion flow to scavengers (some of which is left unused by mammalian and avian scavengers), thereby increasing the troughs, we demonstrate here that the presence of wolves allows for a more predictable and reliable food supply to scavengers.

Winter carrion is crucial to the growth and fitness of many species in the Northern Rockies. As many as 31 species have been documented to feed on wolf kills (P. Paquet, personal communication), but grizzly bears (Ursus arctos), black bears (Ursus americanus), coyotes (Canis latrans), bald eagles (Haliaeetus leucocephalus), golden eagles (Aquila chrysaetos), ravens (Corvus corax) and magpies (Pica pica) are the most common visitors at wolf kills in Yellowstone. Coyotes are highly dependent on winter scavenge (Crabtree and Sheldon, 1999) and they have been shown to track wolves to their kill-sites and to feed despite a high risk of predation by wolves (Paquet, 1992). In addition, Crabtree and Sheldon (1999) found that additional elk carrion increased coyote litter size and pup survival. Raven reproduction has been tied to the availability of winter carrion (Newton et al., 1982) and they have been shown to adopt a foraging strategy of following wolves to locate their kills (Stahler et al., 2002). Grizzly bears also depend on spring carrion and occasionally forego hibernation altogether in Glacier National Park, Montana in favor of scavenging wolf kills (D. Boyd, personal communication).

As wolf and elk populations grow and contract, carrion subsidies to scavengers may change both in abundance and temporal distribution (Wilmers et al., 2003a). Here we simulate the population dynamics of northern Yellowstone elk and the corresponding accrual of carrion across the year in the absence of wolves. Elk mortality is driven by winter environmental conditions and density dependence. We then add wolf harvest to the model and explore how wolves alter the distribution and abundance of carrion over time. We focus on elk because they are the most numerous ungulate in the area (Houston, 1982) and because they account for well over $90 \%$ of the wolf's diet on the northern range (Mech et al., 2001). Further, prey switching is unlikely to occur because of the sheer magnitude of the elk population and the potential alternative, bison, are difficult to kill except under special circumstances (Smith et al., 2000).

Humans also play a major role in provisioning carrion to scavengers. Elk hunters typically dress their catch in the field, leaving behind entrails that become food to scavengers. Unlike wolf predation, however, human hunting is highly localized both in space and time due to restrictions on where and when hunting is allowed. The long-term impacts of hunting are also likely to be different from those of wolves because hunters kill a different segment of the elk population. Wolves kill primarily calves and old individuals (Mech et al., 2001) whereas hunters in the Yellowstone area kill primarily adult cows and usually do not discriminate based on age (T. Lemke, personal communication). We add human hunting to the model and explore scenarios both with and without wolves and with and without human hunting in order to explore the separate and synergistic effects of wolves and hunters on scavenger populations.

Understanding how wolves affect the long-term supply of resource to scavengers in areas with and without hunting is important to conservationists seeking to restore wolf populations in other areas. In addi- 
tion, insight into the interplay between wolf predation and human hunting on carrion supply to scavengers will aid managers formulating hunting policies. The essence of our problem is reduced to a two predator, one prey model that simulates the flow of carrion resource to scavengers. The model allows us to explore conservation and management scenarios (Starfield and Bleloch, 1986) as well as test basic ideas about predator effects on food chains.

\section{Methods}

\subsection{Pre-wolf model}

We use a modified Leslie Matrix model with a monthly time step to simulate the population dynamics of Yellowstone elk and the corresponding accrual of elk carrion as individuals die from one month to the next. Elk calves and old adults experience density-dependent mortality in the winter (Taper and Gogan, 2002) as increasing snow levels concomitantly increase metabolic activity (Parker et al., 1984) and reduce access to food (Houston, 1982). Therefore, we incorporate density dependence into winter survival probabilities of old adults and calves. Males and females are modeled separately to account for the fact that senescence begins much earlier in males than in females (Houston, 1982). Reproduction is limited to females of three years and older and declines when females reach old age (Houston, 1982).

The female components of the elk population over time $t$ are represented by the age class vector $\boldsymbol{x}^{\mathrm{f}}=\left(x_{1}^{\mathrm{f}}, x_{2}^{\mathrm{f}}, \ldots, x_{n}^{\mathrm{f}}\right)^{\prime}$ where the elements, $x_{i}^{\mathrm{f}}$, for $i=1, \ldots, n$, are the number of females in age class $i$. A similar vector $\boldsymbol{x}^{\mathrm{m}}$ defines the male population so that the combined female and male population $\boldsymbol{x}(t)$ at time $t$ satisfies,

$\boldsymbol{x}(t)=\boldsymbol{x}^{\mathrm{f}}(t)+\boldsymbol{x}^{\mathrm{m}}(t)$

where we choose the units of $t$ to be one month. We also let age classes range from 1-year-olds to more than 20-year-olds, i.e. $n=20$, in order to account for the full age range of Northern Yellowstone elk (Houston, 1982). This adds structural realism to the model without increasing the number of parameters to estimate because we keep vital rates unchanged between most ages. Reproduction occurs once a year in June. In non-reproductive months our model takes the form,

$$
\begin{aligned}
& x^{\mathrm{y}}(t+1)=A^{\mathrm{y}}(t) x^{\mathrm{y}}(t) \quad \bmod (t, 12) \neq 6, \\
& \mathrm{y}=\mathrm{f} \text { or } \mathrm{m}
\end{aligned}
$$

where $\boldsymbol{A}^{\mathrm{y}}(t)$ is the transition matrix,

$\boldsymbol{A}^{\mathrm{y}}(t)=\left[\begin{array}{ccccc}P_{1}^{\mathrm{y}} & 0 & \cdot & \cdot & 0 \\ 0 & P_{2}^{\mathrm{y}} & & & \\ \cdot & & \cdot & & \\ \cdot & & & \cdot & \\ 0 & & & & P_{n}^{\mathrm{y}}\end{array}\right]$

and $P_{i}^{\mathrm{y}}$ is the probability that a member of the $i$ th age class survives to the next month. Each June, females reproduce and elk advance age classes such that,

$$
\begin{aligned}
& \boldsymbol{x}^{\mathrm{f}}(t+1)=\left(\boldsymbol{R}^{\mathrm{f}}(t)+\boldsymbol{T}^{\mathrm{f}}(t)\right) \boldsymbol{x}^{\mathrm{f}}(t), \\
& x^{\mathrm{m}}(t+1)=\boldsymbol{T}^{\mathrm{m}}(t) \boldsymbol{x}^{\mathrm{m}}(t)+\boldsymbol{R}^{\mathrm{f}}(t) \boldsymbol{x}^{\mathrm{f}}(t), \\
& \bmod (1,12)=6
\end{aligned}
$$

where the reproductive, $\boldsymbol{R}^{\mathrm{y}}$, and the transition, $\boldsymbol{T}^{\mathrm{y}}$, matrices have the form,

$$
\boldsymbol{R}^{\mathrm{f}}(t)=\left[\begin{array}{ccccc}
0 & 0 & F_{3} & \cdots & F_{n} \\
\cdot & \cdot & & & \\
\cdot & & \cdot & & \\
\cdot & & & \cdot & \\
0 & & & & 0
\end{array}\right]
$$

and,

$$
\boldsymbol{T}^{\mathrm{y}}(t)=\left[\begin{array}{ccccc}
0 & \cdot & \cdot & \cdot & 0 \\
G_{1}^{\mathrm{y}} & & & & \cdot \\
& \cdot & & & \cdot \\
& & \cdot & & \cdot \\
& & & G_{n-1}^{\mathrm{y}} & 0
\end{array}\right]
$$

In these matrices, $F_{i}$ is the fecundity of females and $G_{i}^{\mathrm{y}}$ is the probability of advancing to the next age class. Female elk in Yellowstone are highly productive in their younger years but become substantially less fecund in old age (Houston, 1982). As such, we set fecundity for prime-aged females $F_{i=3, \ldots, \alpha-1}=F_{\max }$ and the fecundity for old females $F_{i=\alpha, \ldots, n}=F_{\min }$. We assume a 50:50 birth sex ratio so that $F_{i}$ represents half of the total value.

Senescence begins in the winter of females' $\alpha$ th year and males' $\beta$ th year. In each subsequent age class, 
an increasing proportion $\gamma_{i}^{\pi}$, where $\pi=\alpha$ or $\beta$, become part of the senescent class. Guided by mortality curves published by Houston (1982), we increase $\gamma_{i}^{\pi}$ quadratically with age such that

$\gamma_{i}^{\pi}=-0.025(i-\pi+1)^{2}+1, \quad$ for $i=\pi, \ldots, n$

Density dependence is incorporated into the survival terms of calves and senescent adults as follows. Let $V$ be the snow-water-equivalent (which serves as a proxy for snow depth that has been found to be strongly correlated with elk mortality (Gese et al., 1996)), B the biomass of the elk population, $P_{\max }$ the maximum survival probability, $\lambda$ the half saturation level and $s$ the shape parameter. Survival probabilities for calves and old adults during the winter time are then described by the function,

$$
\begin{array}{ll}
P_{i}^{\mathrm{f}}=p_{\max }^{\mathrm{f}}, & i=2, \ldots, \alpha-1 \\
P_{i}^{\mathrm{m}}=p_{\max }^{\mathrm{m}}, \mathrm{y} & i=2, \ldots, \beta-1 \\
P_{i}^{\mathrm{y}}=\frac{p_{\max }(\lambda)^{\mathrm{s}}}{(\lambda)^{\mathrm{s}}+\left(V(t)^{2} B(t)^{\mathrm{s}}\right)}, & \text { for } i=1 \text { and } \alpha, \ldots, n \\
& \begin{array}{l}
\text { for females; for } i=1 \\
\text { and } \beta, \ldots, n \text { for males }
\end{array}
\end{array}
$$

This function has the properties that as elk biomass or snow-water-equivalent increase, survival decreases (Fig. 1). The half saturation parameter $\lambda$ sets the point at which maximum survival is reduced by half. The shape parameter $\lambda$ describes the abruptness of the onset of density dependence (Fig. 1) (Getz, 1996). When $s$ is low, density dependence sets in gradually as biomass and snow-water-equivalent increase. As $s$ increases the function approaches a step function where density dependence sets in abruptly as $V^{2} B$ increases. We assume that survival decreases in proportion to the square of snow-water-equivalent because previous studies have indicated that as snow-water-equivalent increases, elk metabolism increases quadratically (Parker et al., 1984). In our simulations we use real snow-water-equivalent values collected on the Northern Range from 1949 to 2001 (Farnes et al., 1999). To do so, we choose a year at random from 1949 to 2001. Monthly snow-water-equivalent values from that year are then assigned to the model corresponding to each winter (December through April) month. Stochasticity is thus introduced into the model via $V$.

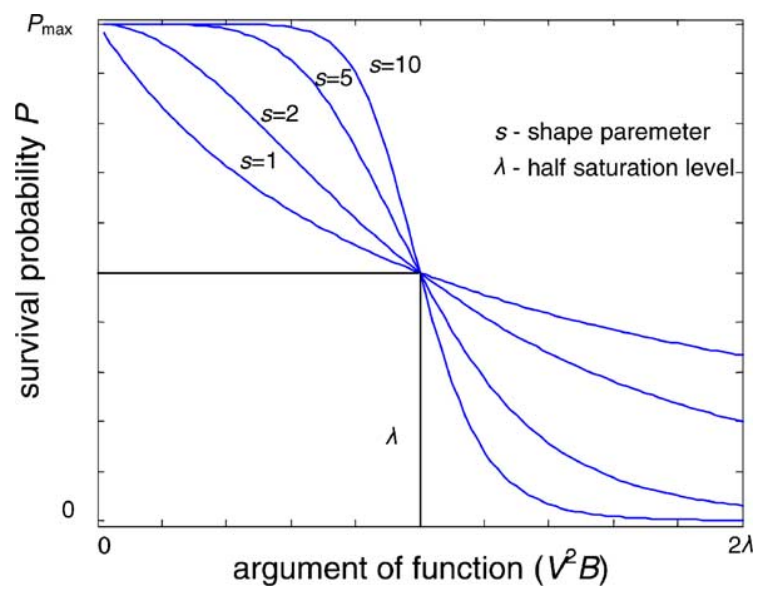

Fig. 1. The sigmoid elk density-dependence function as defined in Eq. (8). Increasing the shape parameter, $s$, increases the abruptness of density dependence onset.

The biomass of the entire elk population is given by

$B(t)=\sum_{i=1}^{n} x_{i}^{\mathrm{f}}(t) \omega_{i}^{\mathrm{f}}(t)+x_{i}^{\mathrm{m}}(t) \omega_{i}^{\mathrm{m}}(t)$

where $\omega_{i}^{\mathrm{f}}$ and $\omega_{i}^{\mathrm{m}}$ are the average monthly weights (in $\mathrm{kg}$ ) of females and males for age $i$ taken from an elk growth model (Murphy et al., 1997). Individuals that do not survive from one month to the next enter the carrion pool for that month. The amount of carrion that accumulates in the months without reproduction (in $\mathrm{kg}$ ) is given by

$$
\begin{aligned}
C(t+1)= & \sum_{i=1}^{n}\left(x_{i}^{\mathrm{f}}(t)-x_{i}^{\mathrm{f}}(t+1)\right) \omega_{i}^{\mathrm{f}}(t)+\left(x_{i}^{\mathrm{m}}(t)\right. \\
& \left.-x_{i}^{\mathrm{m}}(t+1)\right) \omega_{i}^{\mathrm{m}}(t), \quad \bmod (t, 12) \neq 6
\end{aligned}
$$

and,

$$
\begin{aligned}
C(t+1)= & \left(0.5\left(\sum_{i=3}^{\alpha-1} x_{i}^{\mathrm{f}}(t) F_{\max }+\sum_{i=\alpha}^{n} x_{i}^{\mathrm{f}}(t) F_{\min }\right)\right. \\
& \left.-x_{1}^{\mathrm{f}}(t+1)\right) \omega_{1}^{\mathrm{f}}+\left(0 . 5 \left(\sum_{i=3}^{\alpha-1} x_{i}^{\mathrm{f}}(t) F_{\max }\right.\right. \\
& \left.\left.+\sum_{i=\alpha}^{n} x_{i}^{\mathrm{f}}(t) F_{\text {min }}\right)-x_{1}^{\beta}(t+1)\right) \omega_{1}^{\mathrm{m}}
\end{aligned}
$$




$$
\begin{aligned}
& +\sum_{i=1}^{n}\left(x_{i}^{\mathrm{f}}(t)-x_{i+1}^{\mathrm{f}}(t+1)\right) \omega_{i+1}^{\mathrm{f}}(t) \\
& +\left(x_{i}^{\mathrm{m}}(t)-x_{i+1}^{\mathrm{m}}(t+1)\right) \omega_{i+1}^{\mathrm{m}}(t), \\
& \quad \bmod (t, 12)=6
\end{aligned}
$$

in June to account for the effect of reproduction and age class advancement on carrion accrual.

\subsection{Adding wolves}

We incorporate dynamics into the wolf population to explore the relationship between scavenge provision and elk population size as the wolf population responds dynamically to changes in its prey base. Wolves are born at the beginning of April each year, exceeds their energetic requirement (allowing for losses to scavengers), then survival is at its maximum. However, if the harvest is less than this energetic requirement, then resources go into deficit, $D$, and survival begins to decline as a function of the amount of this deficit per wolf. To account for the reduced requirements of pups, we define the relative number of wolves, $W$, where pups are discounted by $a(t)$, $(0<a(t)<1)$ because they are smaller and require less resources than adults, as

$$
W(t)=a(t) w_{1}(t)+w_{2}(t)+w_{3}(t)
$$

The dependence of $a$ on $t$ arises because we assume that pup growth is a linear function of time with pups starting out at a fraction $a_{0}$ of full growth and becoming full-grown at 10 months, viz.

$$
\begin{array}{ll}
a(t)=a_{0}+\left(\frac{1-a_{0}}{10}\right)(t-4), & \bmod (t, 12)=4,5=12,1 \\
a(t)=1, & \bmod (t, 12)=2,3
\end{array}
$$

and reach sexual maturity at two years of age. As such we define three age classes of wolves: pups $\left(w_{1}\right.$ : 0 to 1-year-olds), juveniles ( $w_{2}: 1$ - to 2-year-olds), and adults $\left(w_{3}\right.$ : $>2$-year-olds) where the minimum breeding age is set at 22 months (Haight et al., 1998). If we let $S_{j}, j=1,2,3$, represent the survivorship of the $j$ th age class, and $L_{3}$ be the fecundity of adults then the change in wolf population $w$ over the interval $[t, t+1]$ satisfies,

$\boldsymbol{w}(t+1)=\boldsymbol{M}(t) \boldsymbol{w}(t), \quad t=0,1,2, \ldots$ (months)

where $\boldsymbol{M}(t)$ is the transition matrix given by,

$$
\boldsymbol{M}(t)=\left[\begin{array}{ccc}
0 & 0 & L_{3} \\
S_{1} & 0 & 0 \\
0 & S_{2} & S_{3}
\end{array}\right], \quad \text { for } \bmod (t, 12)=14
$$

$$
\boldsymbol{M}(t)=\left[\begin{array}{ccc}
S_{1} & 0 & 0 \\
0 & S_{2} & 0 \\
0 & 0 & S_{3}
\end{array}\right], \quad \text { for } \bmod (t, 12) \neq 4
$$

Wolf survivorship is assumed to depend on the quantity of prey resource. If the harvest of elk by wolves
If we let $S_{\max }$ be the maximum survival probability, $v$ the half saturation constant, $\zeta$ the shape parameter, $E$ the energetic requirement per wolf per month, and $K(t)$ the kill-rate per wolf per month (in $\mathrm{kg}$ ), survivorship then becomes,

$S_{j}(t)=S_{\max }\left(1-\frac{(D(t))^{\varsigma}}{v_{j}^{\varsigma}+(D(t))^{\varsigma}}\right)$

where

$$
\begin{array}{ll}
D(t)=0, & \text { for } E \leq K(t) \\
D(t)=E-K(t), & \text { for } E>K(t)
\end{array}
$$

While wolves may kill an occasional prime-aged elk, they are primarily dependent on the old and young for their sustenance (Peterson et al., 1984, Mech et al., 2001). Hence the biomass of vulnerable prey $B_{\mathrm{v}}$ is the sum of calves and senescing adults given by,

$$
\begin{aligned}
B_{\mathrm{v}}(t)= & \omega_{1}^{\mathrm{m}}(t) x_{1}^{\mathrm{m}}(t)+\omega_{1}^{\mathrm{f}}(t) x_{1}^{\mathrm{f}}(t)+\sum_{i=\alpha}^{n} \gamma_{i} \omega_{i}^{\mathrm{f}}(t) x_{i}^{\mathrm{f}}(t) \\
& +\sum_{i=\beta}^{n} \gamma_{i} \omega_{i}^{\mathrm{m}}(t) x_{i}^{\mathrm{m}}(t)
\end{aligned}
$$

The functional form of wolf kill-rate has recently been shown to depend on the ratio of the number of wolves to the number of prey (Vucetich et al., 2002). 
Though Vucetich et al. found that a ratio-dependent function was among the best fits to the data (Pitcairn et al., 1990), it still only explained $33 \%$ of the variance. While many factors may contribute to explaining the remaining variance, we believe that one improvement would be to make kill-rate depend on the ratio of wolves to the biomass of vulnerable prey rather than total prey numbers. This more accurately represents the segment of the prey population vulnerable to predation and the actual availability to wolves of the prey resource. Kill-rate per wolf in our model then is assumed to be a function of the ratio of the number of wolves to biomass of vulnerable prey given by

$$
K(t)=\frac{K_{\max } \mu^{\xi}}{\mu^{\xi}+\left(W(t) / B_{\mathrm{V}}(t)\right)^{\xi}}
$$

where $K_{\max }$ is the maximum kill-rate per wolf, $\mu$ is the half saturation constant and $\xi$ is the shape parameter (Fig. 2).

If the elements $h_{i}^{\mathrm{y}}$ of $\boldsymbol{h}^{\mathrm{y}}, i=1, \ldots, n$ and $\mathrm{y}=\mathrm{f}$ or $\mathrm{m}$, represent the number of additional elk harvested each month from age class $i$ and gender class y, that would not have died in the absence of wolves, then the change in elk population over time with wolf harvesting is given by

$$
\begin{aligned}
& \boldsymbol{x}^{\mathrm{y}}(t+1)=\boldsymbol{A}^{\mathrm{y}}(t) \boldsymbol{x}^{\mathrm{y}}(t)-\boldsymbol{h}^{\mathrm{y}}(t), \\
& \quad \text { for } \bmod (t, 12) \neq 6
\end{aligned}
$$

and

$$
\begin{aligned}
& \boldsymbol{x}^{\mathrm{f}}(t+1)=\left(\boldsymbol{R}^{\mathrm{f}}(t)+\boldsymbol{T}^{\mathrm{f}}(t)\right) \boldsymbol{x}^{\mathrm{f}}(t)-\boldsymbol{h}^{\mathrm{f}}(t), \\
& \quad \boldsymbol{x}^{\mathrm{m}}(t+1)=\boldsymbol{T}^{\mathrm{m}}(t) \boldsymbol{x}^{\mathrm{m}}(t)+\boldsymbol{R}^{\mathrm{f}}(t) \boldsymbol{x}^{\mathrm{f}}(t)-\boldsymbol{h}^{\mathrm{m}}(t), \\
& \quad \text { for } \bmod (1,12)=6
\end{aligned}
$$

Wolf predation is assumed to be additive during times of the year when natural elk mortality would be less than wolf demands and to be compensatory during times when natural mortality would have exceeded wolf demands. The total take, $H$, of elk biomass killed by wolves is given by

$$
H(t)=K(t) W(t)
$$

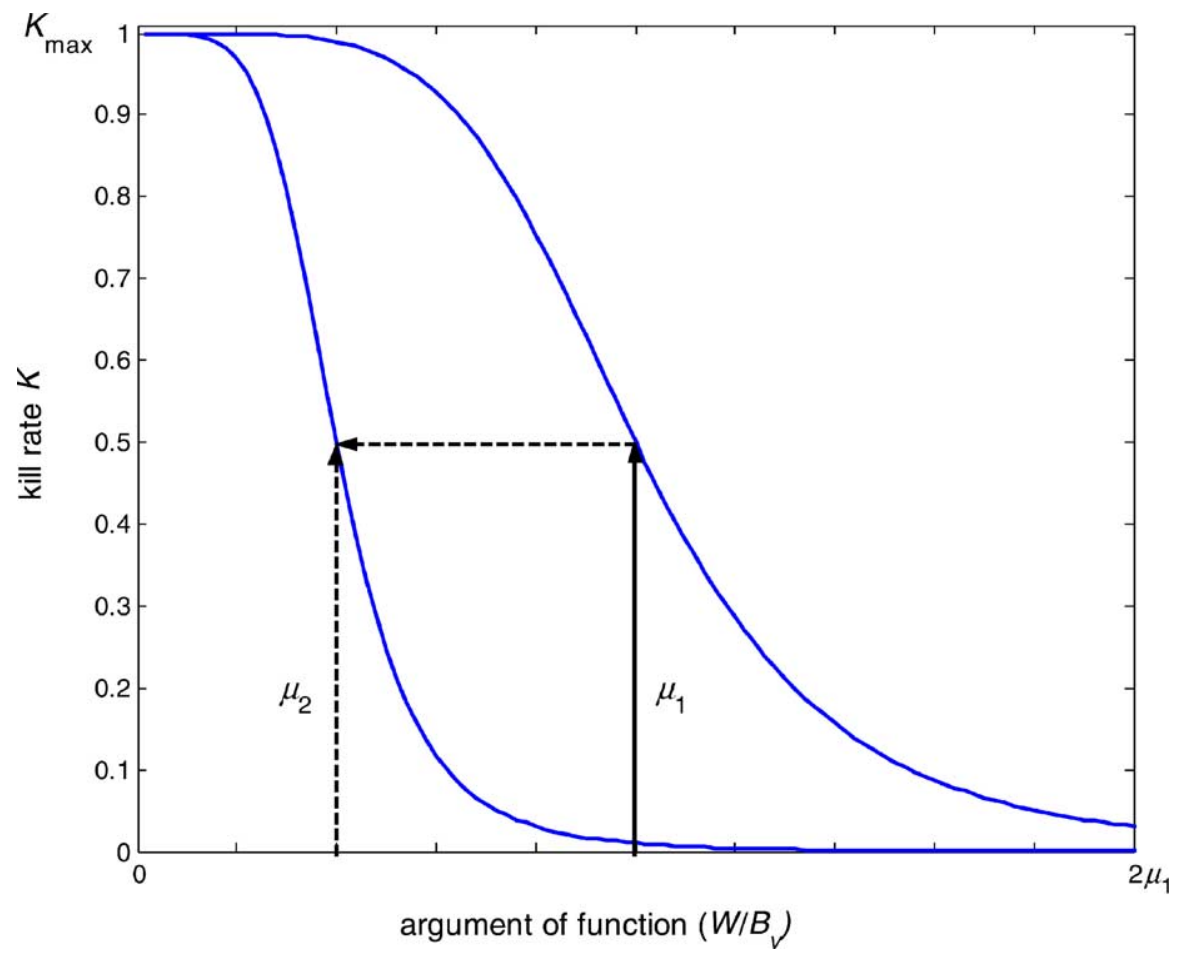

Fig. 2. Pictorial representation of the wolf kill-rate function as defined in Eq. (19). As the half saturation level decreases from $\mu_{1}$ to $\mu_{2}$, wolf kill-rate $K$ declines at lower ratios of wolves to elk. 
During certain times of the year, some or all of the carrion produced by wolves may have otherwise died. Hence we express the additional take, $H_{\mathrm{a}}$, of elk biomass when wolf predation is additive by

$$
\begin{aligned}
& H_{\mathrm{a}}(t) \equiv \sum_{i=1}^{n} h_{i}^{\mathrm{y}}=K(t) W(t)-C(t), \\
& \quad \text { for } K(t) W(t)>C(t)
\end{aligned}
$$

We then convert elk biomass to numbers by dividing the total carrion in each age class by the average weight of an individual in that age class and month of the year. Calves and senescing adults are killed in proportion to their abundance in the population. The oldest senescent adults are harvested first, followed by the next oldest and so on.

The amount of carrion available each month to scavengers $C_{\mathrm{S}}$ is simply total elk mortality less that which wolves consume: that is,

$C_{\mathrm{S}}(t)=C(t)+\sum_{i=1}^{n} h_{i}^{\mathrm{y}}(t)-E W(t)$

\subsection{Hunter harvest}

Human hunting of the Northern Yellowstone elk herd occurs north of the park on national forest land as animals leave the park in the winter. There are two hunts: the early hunt from September to December which is largely unregulated and the late hunt from January to February for which quotas are set. The late hunt accounts for the bulk of the total take for the entire winter hunting season (over $80 \%$ in some years) (Lemke et al., 1998) as elk are at lower elevations and less dispersed on the landscape. Late hunt quotas are set in order to regulate elk numbers and to provide sustainable public recreation (Lemke et al., 1998). Hunting permit quotas are set using adaptive harvest management (AHM) guidelines which take into account the number of elk migrating north of the park and hunter success rates (T. Lemke, personal communication). Approximately $95 \%$ of the permits issued are antler-less. So the majority of hunters take adult cows.

We incorporate hunting into the model by assuming that all hunting occurs in the late winter period. As the number of elk wintering north of the park is likely to correlate with population size (in addition to winter severity), we simply set the hunting level, $\theta$, as a percentage of the total population. We then specify the proportion of kills that are cows $\rho_{\text {cow }}$. Calves are harvested at a fixed low background proportional rate $\rho_{\text {calf }}$. The proportion of bulls harvested is then $\rho_{\text {bull }}=$ $1-\rho_{\text {calf }}-\rho_{\text {cow }}$. The number of individuals in each age class harvested is proportional to the abundance of that age class in the population. Based on weights of hunter gut piles (Wilmers et al., 2003a), we assume that $14 \%$ of each hunter kill becomes food for scavengers.

Actual quotas set by managers in the Yellowstone Ecosystem are based on count data and hunter success rates which have a degree of error in them. In addition, other factors affect the quota such as weather conditions and public comment. In this study, however, our goal is to understand some of the basic biological interactions between hunters and scavengers rather than to precisely model the Northern Yellowstone elk hunt.

\subsection{Simulations and sensitivity analysis}

The model was coded in Matlab 6.0.1 (Mathsoft ${ }^{\mathrm{TM}}$ ). We ran the model for 500 years with monthly time steps and deleted the first 100 years of data in order to remove the transient effects of initial conditions. We then collected basic descriptive statistics on carrion levels within and across years. In order to quantify the spread of carrion by month $j$ across a single year we calculated its normalized Shannon-Weaver diversity number, $\Phi$, given by,

$\Phi=-\frac{1}{\log (12)} \sum_{i=j}^{12} Q_{j} \ln \left(Q_{j}\right)$

where,

$Q_{j}=\frac{C_{j}}{\sum_{j=1}^{12} C_{j}}$

This is an index ranging between zero and one, which relates the evenness of the carrion spread across the year. A Shannon diversity number of 0 indicates that all the carrion occurs in one month of the year whereas a value of 1 indicates that the carrion is evenly distributed across each month of the year.

Sensitivity analyses were conducted using Monte Carlo methods to assess the relative effects of several parameters on model statistics (Wisdom and Mills, 1997; Wisdom et al., 2000; Cross and Beissinger, 
Table 1

Parameter descriptions and data ranges

\begin{tabular}{|c|c|c|c|c|}
\hline Function & Parameter & Description & Range & Source \\
\hline Elk & $\begin{array}{l}\alpha \\
\beta \\
s \\
\lambda \\
F_{\max } \\
F_{\min } \\
P_{1}^{\mathrm{y}}(6)\end{array}$ & $\begin{array}{l}\text { Onset of female senescence } \\
\text { Onset of male senescence } \\
\text { Abruptness parameter } \\
\text { Half saturation level } \\
\text { Fecundity of prime-aged females } \\
\text { Fecundity of senescing females } \\
\text { Summer calf survival }\end{array}$ & $\begin{array}{l}10-13 \text { years } \\
4-7 \text { years } \\
1-2 \\
3-5 \times 10^{6} \mathrm{~kg} \\
0.6-0.85 \\
0.4-0.6 \\
0.5-0.8\end{array}$ & $\begin{array}{l}\text { Houston }(1982) \\
\text { Houston }(1982) \\
\text { Getz }(1996)^{\mathrm{a}} \\
-^{\mathrm{b}} \\
\text { Houston (1982) } \\
\text { Houston (1982) } \\
\text { Singer et al. (1997) }\end{array}$ \\
\hline Hunt & $\begin{array}{l}\theta \\
\rho_{\text {cow }}\end{array}$ & $\begin{array}{l}\text { Proportion of population to harvest } \\
\text { Proportion of cows to harvest }\end{array}$ & $\begin{array}{l}0.025-0.06 \\
0.5-0.95\end{array}$ & $--^{c}$ \\
\hline Wolf kill-rate & $\begin{array}{l}\mu \\
\xi\end{array}$ & $\begin{array}{l}\text { Half saturation } \\
\text { Abruptness parameter }\end{array}$ & $\begin{array}{l}0.00003-0.0003 \\
1-4\end{array}$ & $\begin{array}{l}\text { Fuller (1989), Vucetich et al. }(2002)^{\mathrm{d}} \\
\text { Getz (1996) }\end{array}$ \\
\hline Wolf survival & $\begin{array}{l}\zeta \\
v_{1} \\
v_{2,3} \\
\mathrm{E} \\
L\end{array}$ & $\begin{array}{l}\text { Abruptness parameter } \\
\text { Half saturation of pups } \\
\text { Half saturation of juveniles and adults } \\
\text { Energetic requirement } \\
\text { Fecundity }\end{array}$ & $\begin{array}{l}1-2 \\
50-100 \\
100-200 \\
1-5 \mathrm{~kg} \text { per day } \\
1-6\end{array}$ & $\begin{array}{l}\text { Getz }(1996)^{\mathrm{a}} \\
\text { e }_{\mathrm{e}} \\
\text { e }^{\mathrm{F}} \\
\text { Fuller }(1989) \\
\text { Mech }(1970)\end{array}$ \\
\hline
\end{tabular}

${ }^{a}$ Because of the extreme sensitivity to this parameter, we confined it to this narrow range. In addition, for the reasons discussed in Getz (1996), this parameter is likely to be small (i.e. <2) for mammals with large storage (fat) capabilities (elk) or for territorial animals (wolves).

b We chose half saturation levels that yielded mean elk numbers of 15,000-25,000 elk in the pre-wolf model.

${ }^{c}$ Ranges were chosen to test the effects of different management scenarios.

d We converted data given in numbers to biomass in order to estimate $\mu$.

e We chose ranges based on intimate knowledge of the system that were larger than they probably are. This would tend to overestimate the sensitivity of this parameter.

2001). Specifically, for each model, 1000 random parameter sets were created by choosing model specific parameter sets from uniform distributions bounded by the values shown in Table 1. Parameter ranges were either estimated from previously published work or chosen a priori in order to test the effect of different management scenarios (such as the case with hunt level). Where specific ranges were not given in published work, we estimated a range based on our best understanding of the biology. Each parameter set was used to run the model once, for a total of 1000 runs. Mean yearly carrion levels and Shannon diversity numbers for the years 101-500 were recorded for each run and used as the dependent variable in linear regressions in which the model parameters were the explanatory variables. Model parameters were ranked according to $r^{2}$ values in order to determine which ones explained the most variance in model output statistics (Wisdom and Mills, 1997; Wisdom et al., 2000; Cross and Beissinger, 2001). The larger the range in a parameter, the higher its $r^{2}$ may become. As such we generally erred on the side of caution, choosing larger rather than smaller ranges so that our bias would be to- wards overestimating the sensitivity of an output variable to the parameter.

Each model typology was thus analyzed for parameter sensitivities. Model statistics from the pre-wolf model were then compared to parallel statistics for each successive post-wolf model by running each model through its most sensitive parameters.

\section{Results}

\subsection{Carrion accrual and diversity}

The pre-wolf model generates a changing elk population over time with a corresponding accrual of elk carrion across the year (Fig. 3A). Within year fluctuations in biomass largely reflect the changing weight of the elk population as they gain weight during the summer and lose it during the winter (Fig. 3B). Between year changes in elk biomass reflect changes in elk number or age structure. Carrion levels during the summer months are low and begin to accumulate during the winter months as snow levels increase and elk 


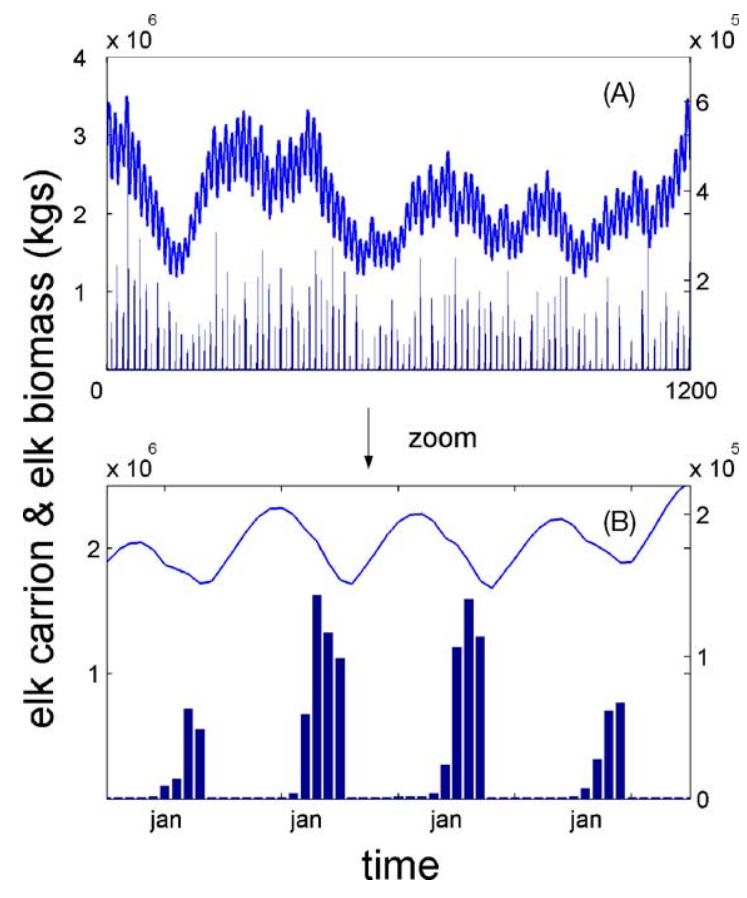

Fig. 3. Output of a sample run of the model for average parameter values taken from Table 1: (A) pre-wolf-elk biomass over 100 years (line with scale on left axes) and corresponding elk carrion (bars with scale on right axes); (B) 4 year subset of the full run.

weaken and die. Though the distribution of elk carrion varies from year to year depending on the snow pack and population size, the general pattern is for carrion to build during the course of the winter and peak near March (Fig. 3B and 4A). In addition the total abundance of elk carrion roughly follows the size of the elk population. In a single run of the model, using mean values of each parameter, average winter snow depth accounts for 53\%, and elk number accounts for $40 \%$ of the variance in mean yearly carrion, respectively.

The addition of wolves to the model results in a reduction of the amount of late winter carrion, but extends the availability of carrion to early winter (November and December) and other times of year when carrion would not previously have been available in large quantities (Fig. 4). In addition, wolves reduce the year to year variance in carrion availability (Fig. 4). By killing continuously throughout the year, wolves provide carrion at times that it would not have been available otherwise and also reduce the pool of weak animals entering the winter. As a result, carrion is less plentiful in severe winters but more abundant in mild ones.

\subsection{Abruptness parameter}

The shape parameter of density-dependent elk survival $s$ (Eq. (8)) accounts for $98 \%$ of the variance without wolves in the distribution of carrion across the year as measured by our statistic $\Phi$ (Table 2; Fig. 5A). As $s$ increases, the carrion diversity index $\Phi$ decreases which implies that the more abrupt the onset of density-dependent mortality, the more aggregated elk deaths become. Mean yearly carrion abundance and elk numbers were sensitive to changes in the onset of female senescence $\alpha$, female fecundity $F_{\max }$, shape parameter $s$ and the half saturation constant $h$ (Table 2). The sensitivity results of carrion abundance

Table 2

The $r^{2}$ values of parameters with respect to three indices, mean Shannon diversity index $\bar{\Phi}$, mean elk number $\bar{x}$, and mean carrion $\bar{C}$ obtained from Monte Carlo simulations

\begin{tabular}{|c|c|c|c|c|}
\hline \multirow[t]{2}{*}{ Model } & \multirow[t]{2}{*}{ Parameter } & \multicolumn{3}{|l|}{$r^{2}$} \\
\hline & & $\bar{\Phi}$ & $\bar{x}$ & $\bar{C}$ \\
\hline \multirow[t]{4}{*}{ Pre-wolf no hunt } & $\alpha$ & $-^{\mathrm{a}}$ & 0.16 & 0.19 \\
\hline & $F_{\max }$ & - & 0.08 & 0.20 \\
\hline & $s$ & 0.98 & 0.29 & 0.21 \\
\hline & $\lambda$ & - & 0.45 & 0.34 \\
\hline \multirow[t]{6}{*}{ Pre-wolf with hunt } & $\theta$ & - & 0.08 & 0.08 \\
\hline & $F_{\max }$ & - & 0.06 & 0.11 \\
\hline & $s$ & 0.86 & 0.29 & 0.16 \\
\hline & $\lambda$ & - & 0.19 & 0.10 \\
\hline & $\theta$ & - & 0.16 & 0.27 \\
\hline & $\rho_{\text {cow }}$ & - & 0.15 & 0.16 \\
\hline \multirow[t]{7}{*}{ Post-wolf no hunt } & $\alpha$ & - & 0.12 & 0.10 \\
\hline & $F_{\max }$ & - & 0.07 & 0.09 \\
\hline & $s$ & 0.20 & 0.22 & 0.09 \\
\hline & $\lambda$ & - & 0.25 & 0.12 \\
\hline & $E$ & 0.07 & - & 0.05 \\
\hline & $\mu$ & 0.54 & 0.19 & 0.36 \\
\hline & $\xi$ & 0.07 & - & 0.05 \\
\hline \multirow[t]{8}{*}{ Post-wolf with hunt } & $\alpha$ & - & 0.05 & 0.05 \\
\hline & $F_{\max }$ & - & 0.07 & 0.12 \\
\hline & $s$ & 0.13 & 0.21 & 0.08 \\
\hline & $\lambda$ & - & 0.18 & 0.09 \\
\hline & $E$ & 0.12 & - & - \\
\hline & $\mu$ & 0.51 & 0.11 & 0.24 \\
\hline & $\theta$ & - & 0.17 & 0.27 \\
\hline & $\rho_{\text {cow }}$ & - & 0.14 & 0.15 \\
\hline
\end{tabular}

a We only report results with $r^{2} \geq 0.05$. 


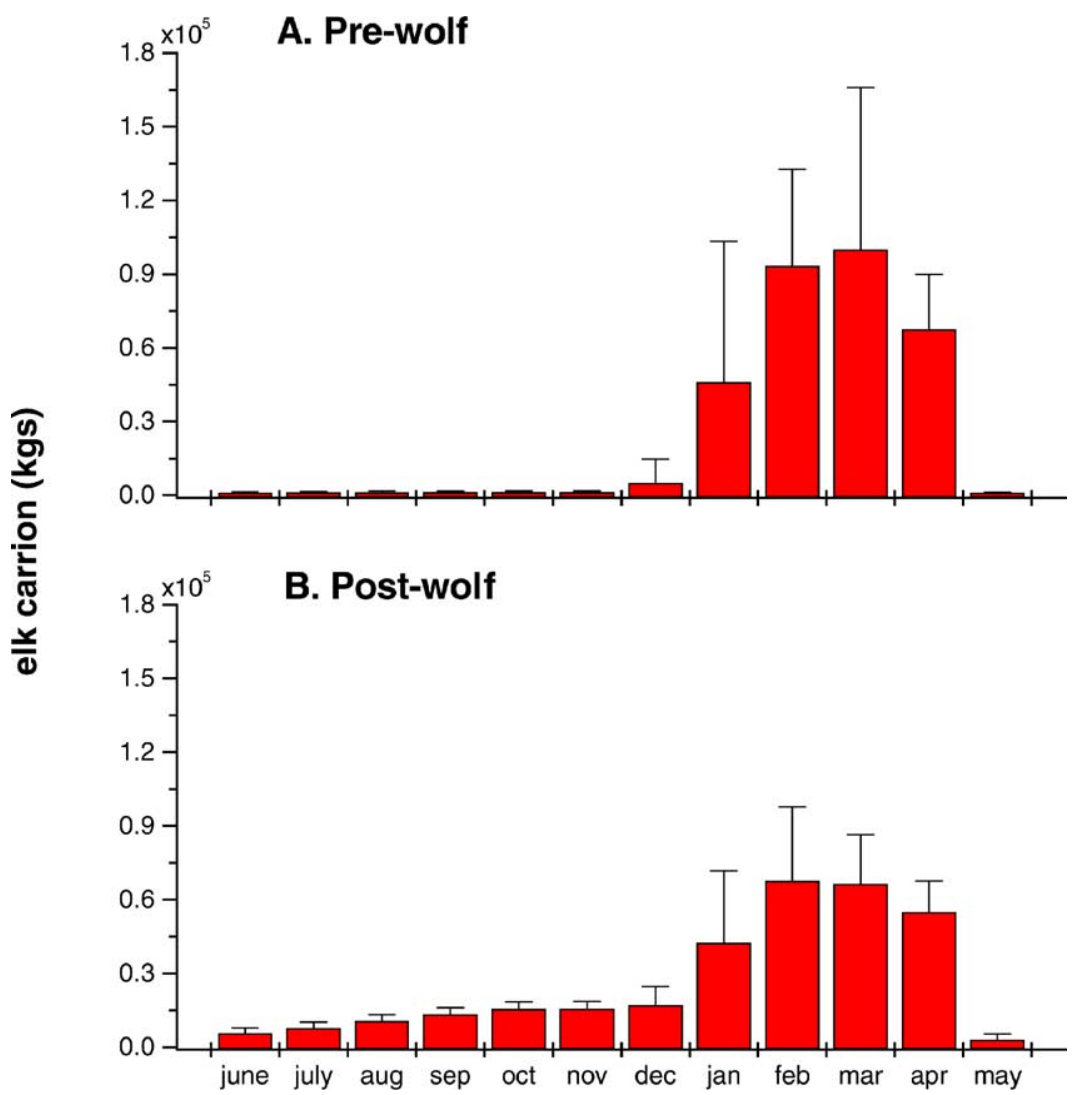

Fig. 4. (A) Average pre-wolf carrion distribution generated from one run of the model with average parameter values taken from Table 1 and the half saturation parameter $\lambda$ tuned so as to generate an average elk population of 17,000 , (B) average post-wolf carrion distribution generated with the same pre-wolf parameter set as in (A) and average post-wolf parameters taken from Table 1 with the wolf kill-rate half saturation parameter $\mu$ tuned so as to generate and average wolf population of 100. Error bars represent one standard deviation.

largely parallel those of elk number (Table 2) because, on average, more elk result in more carrion.

Introducing wolves to the model reduces elk numbers and carrion levels. In addition to the four parameters affecting the sensitivity of these two variables in the pre-wolf model, mean numbers of elk and carrion levels are sensitive to changes in the wolf half-saturation kill-rate $\mu$ (Table 2). As $\mu$ decreases, wolf kill-rate declines at smaller ratios of wolves to prey (Fig. 2) thus allowing the elk population to attain higher average numbers. The fewer wolves there are, the more elk and hence more carrion there is. Conversely, as wolves become more efficient predators and $\mu$ increases, the elk population shrinks and there are more wolves and less total carrion as a result.

Wolves have a large impact on the distribution of carrion throughout the winter. While the carrion di- versity index, $\Phi$, is still sensitive to changes in the elk abruptness parameter $s$, the majority of the variance in $\Phi$ can now be explained by $\mu$ (Table 2). In addition, wolves increase $\Phi$ levels for all values of $\mu$ (Fig. 5B) indicating that the distribution of carrion in the presence of wolves is more evenly spread throughout the year. The effect of $\mu$ on $\Phi$ and mean carrion levels, respectively, are opposed to each other. This implies that as wolves become more efficient predators and hence attain higher population sizes, carrion is more evenly distributed throughout the year, but there is less of it. Conversely, as wolves become less efficient predators and hence attain lower population sizes, total carrion increases but the distribution becomes increasingly skewed towards late winter. With few wolves, elk mortality is primarily driven by winter conditions and density-dependent phenomena result- 


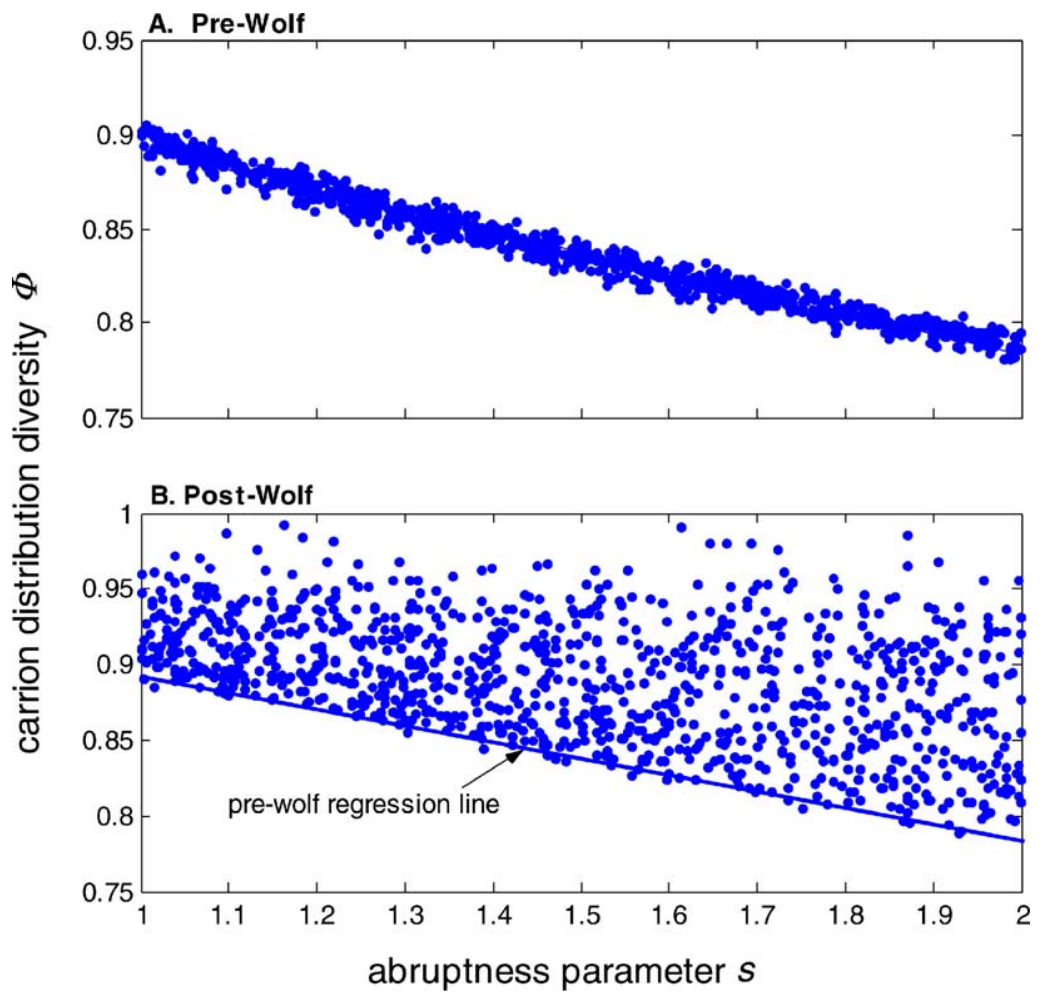

Fig. 5. Carrion diversity index plotted for 1000 runs of the model, choosing parameter values from their uniform distributions (Table 1), except for the abruptness parameter $s$ which has the specific values represented by the $x$-axis: (A) pre-wolf model; (B) post-wolf model with pre-wolf regression line plotted for purposes of comparison.

ing in a pulse of carrion at the end of severe winters. The more wolves there are, the more additive elk mortality there is in early winter and other times of the year (Fig. 4B). This results in a more equitable distribution of carrion throughout the year but less of it because the population of elk is reduced.

\subsection{Hunting}

The addition of hunting to the model also results in lower carrion yields because hunting reduces the elk population. As in the pre-wolf model with no hunting, mean elk numbers and carrion levels are still sensitive to changes in $\alpha, F_{\max }, s$, and $h$ though the effect of these parameters is reduced. Hunt level $\theta$, and the proportion $\rho_{\text {cow }}$ of harvested elk that are cows are now important factors in explaining the variance in population size and carrion abundance (Table 2). The more elk that are hunted or the higher the proportion of adult females harvested, the lower the overall population. Hunting has very little effect on the distribution of carrion across the winter, however. As in the pre-wolf model, $\Phi$ is only affected by changes in the elk abruptness parameter $s$ (Table 2).

The addition of hunting to the post-wolf model largely parallels the effect of the addition of hunting to the pre-wolf model. The elk population and mean carrion levels are reduced with both variables being sensitive to changes in hunt level and the proportion of cows that are harvested. In addition, the distribution of carrion across the year remains sensitive primarily to changes in $\mu$ as in the wolf model without hunting.

Assuming the hunt level $\theta$ remains the same, changes in the proportion $\rho_{\text {cow }}$ of cows harvested can have a large effect on elk population size and hence carrion levels. As an example of this effect, we assume average parameter values from Table 1 and tune $\lambda$ such that the pre-wolf model generates an 

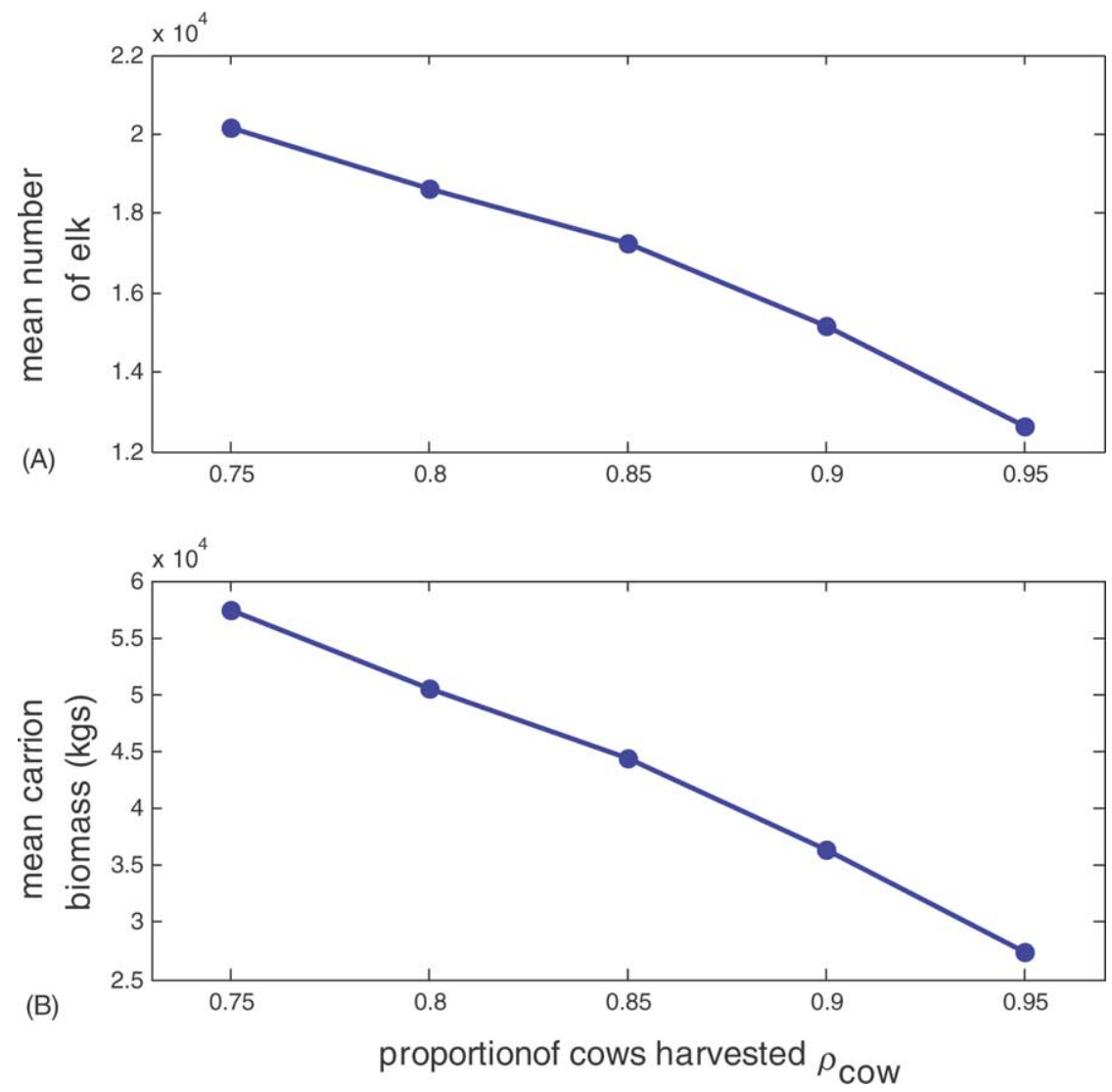

Fig. 6. Comparison of (A) elk numbers and (B) carrion levels under different proportions $\rho_{\text {cow }}$ of cows harvested. We used the same parameter set as those in Fig. 4 with a hunting level $\theta=0.05$ and ran the model for each level of $\rho_{\text {cow }}$ represented on the $x$-axis; $y$-values represent the mean value of elk numbers and carrion levels of each run of the model, respectively.

average elk population of 17,000 individuals with a hunting level $\theta=0.05$ and $\rho_{\text {cow }}=0.95$. If we then add wolves with a half saturation kill-rate $\mu$ tuned such that a mean of 100 wolves persists in the system, the average elk population drops to 13,000. Reducing the proportion of cows harvested by hunters, $10 \%$, to $\rho_{\text {cow }}=0.85$ would restore the elk population to its original 17,000 individuals and hence boost mean carrion levels (Fig. 6).

\subsection{Survivorship}

By selectively preying on old and young elk, wolves cause a decrease in the survivorship of calves and the very old (Fig. 7). By reducing the elk population overall, however, wolves cause an increase in the survivor- ship of individuals that have just begun to senesce (Fig. 7). By reducing the number of elk, wolves mitigate the effect of density-dependent resource competition between elk, causing elk that have just begun to senesce to be better off than they would have been in the absence of wolves. As they get older, however, this effect is overcome by predation by wolves. One effect of these changes in survivorship is that a greater turnover in the elk population occurs. This results in higher carrion yields per elk in the population.

Hunting also changes the shape of the elk survivorship curve (Fig. 7). By hunting cows indiscriminate of age, this lowers the survivorship of adult cows. In addition, by reducing the population and hence the effects of density dependence it also increases the survivorship of calves. 


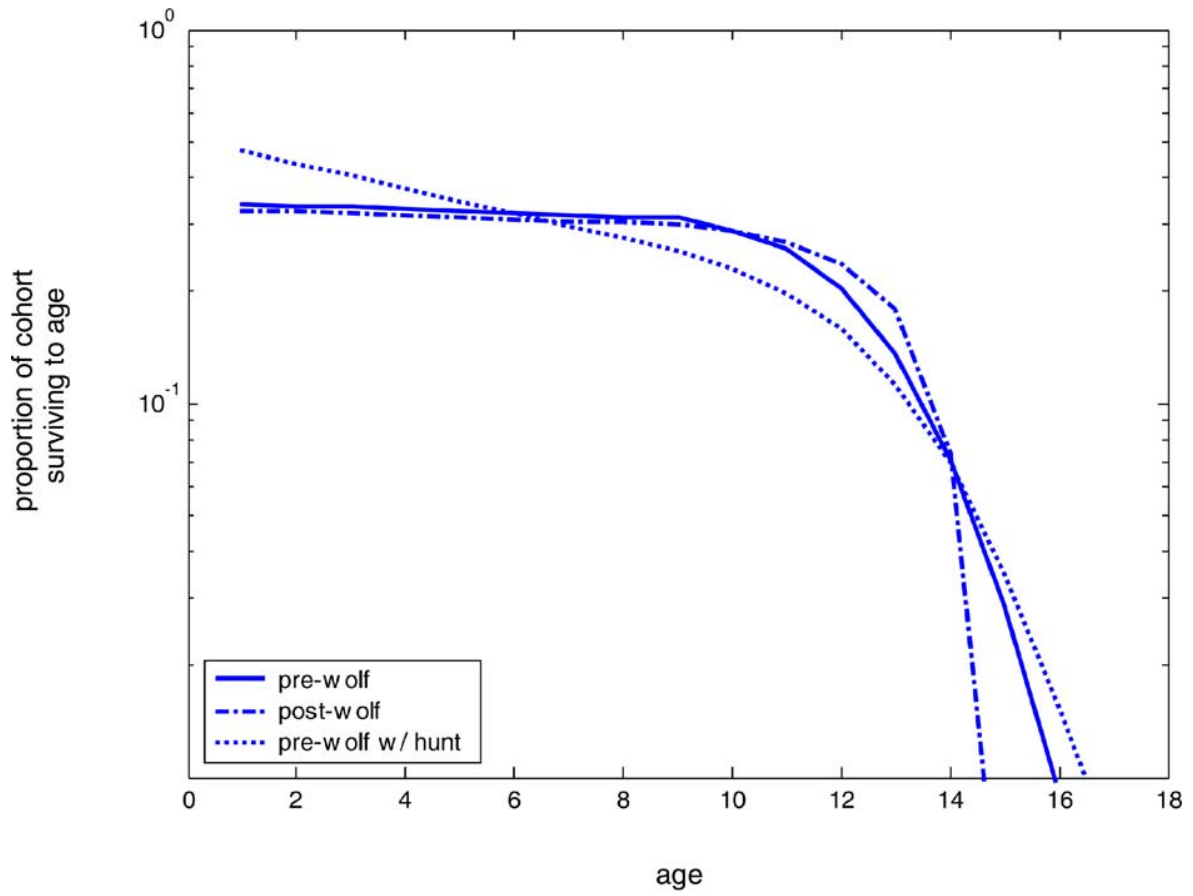

Fig. 7. Comparison of female elk survivorship curves generated by following each cohort through to their deaths. We then take the average survival of each cohort for one run of the model. We use the same parameter sets as those in Fig. 4 with a hunt level $\theta=0.05$ and proportions of cows harvested $\rho_{\text {cow }}=0.95$. Survivorship curves are generated for the pre-wolf, pre-wolf with hunting and post-wolf models.

\section{Discussion}

Elk carrion is a crucial food resource for scavengers. Our model reveals that although wolves reduce the size of the elk population and hence the abundance of elk carrion, they smooth out the temporal distribution of carrion providing carrion throughout the year when previously carrion was only available at the end of winter. In addition, wolves reduce the year to year variance in carrion availability. Whereas prior to wolf reintroduction, carrion would have been plentiful at the end of severe winters and largely absent in mild ones, carrion is now likely to be relatively more plentiful in mild winters and less abundant in severe ones. Since wolf reintroduction, carrion represents a more reliable food resource than in the previous boom and bust cycle. The change in carrion resource availability is likely to affect scavenger species differentially. Small to medium size scavengers with small fat stores are likely to benefit from the more steady supply of carrion. Large scavengers, such as grizzly bears, may experience less of a benefit because they have large fat stores and could thus more easily track the pre-wolf boom and bust scavenge cycle. The fact that carrion is now available in the fall, however, will likely benefit bears going into hibernation by providing a high calorie food prior to denning. Thus, wolves may actually facilitate average population levels of scavengers even though they reduce total annual carrion levels.

The total size of the elk population, and hence abundance of carrion, was found to be sensitive to the half saturation $\lambda$, abruptness $s$, fecundity of prime age females $F_{\max }$ and onset of female senescence $\alpha$ in the elk equations. The parameters $\lambda$ and $s$ control abundance because they control the onset and rapidity of density dependence. The parameters $\alpha$ and $F_{\max }$ are important parameters because they determine the proportion of the population that are prime breeders and how many of these actually give birth. Without wolves, the distribution of carrion across the year, as measured by our diversity statistic $\Phi$, was only sensitive to $s$. Given a high value of $s$, density dependence is absent until a critical density is obtained at which point survivorship drops precipitously and a spike in carrion level 
occurs. Conversely, for a low value of $s$, the effects of density dependence set in relatively slowly and carrion accumulates at a lower rate over a longer period. The addition of wolves to the model reduces the dependence of $\Phi$ on $s$ and results in greater variance in the distribution (Fig. 5B). This is due to the effect of wolves decreasing the elk population and hence the effects of density dependence on the herd. The degree to which wolves reduce the elk population and hence carrion abundance is primarily dependent on the fit of the wolf-kill-rate function. Fitting this function with Yellowstone data in order to estimate parameters, in particular the half-saturation parameter $\mu$ will be crucial in determining the ultimate equilibrium levels of wolves, elk and carrion. While the kill-rate per wolf is currently being estimated each year in Yellowstone (Mech et al., 2001), fitting Eq. (19) will require a longer term data set.

Though wolves reduce the overall abundance of elk carrion by reducing the elk population, this is partially mitigated by the effect of wolves on the turnover of the elk population. By wolves preying selectively on old animals and thus reducing the average age of the elk population, elk productivity is increased. This in turn leads to increased carrion yield per elk in the population. This is akin to the findings of research on herbivores increasing the productivity of the plants they feed on by removing dead tissue thus allowing remaining plant tissue better access to sunlight for photosynthetic activity (McNaughton, 1984).

An interesting consequence of the selective predation by wolves is that they actually increase the survival probabilities of early senescing elk. Arguments for predators strengthening prey populations have generally drawn on evolutionary arguments of predators selecting less fit individuals and thus weeding those genes out of the population (Krebs and Davies, 1981). Here we present a possible ecological explanation for the same phenomenon: that by reducing the effect of density-dependent resource competition among elk, those that remain, even some of the older animals, are better fed and healthier as a result.

Hunting exerts a strong downward pressure on the elk population when cows are the prime target of hunters. Elk population numbers are especially sensitive to the survival of prime-aged cows because they are responsible for the future reproductive yield of the population. An important goal of the late winter elk hunt has been to regulate the population migrating north of the park in order to avoid conflicts with livestock operations in Paradise Valley, maintain the long-term diversity and productivity of winter range vegetation and avoid the transmission of brucellosis from elk to domestic livestock (Lemke et al., 1998). Additionally, the hunt serves as a popular recreational activity important to the winter economy of the local area. Our model reveals that in addition to these concerns, hunt intensity affects the supply of carrion to scavengers. In the short term, a large hunt may provide a localized boom in carrion to scavengers, but in the long term, large hunts suppress the elk population and reduce overall carrion availability from wolf kills which are available throughout the year and throughout the northern range. With the addition of wolves to the ecosystem, the elk herd is likely to experience a reduction in equilibrium population levels. This potentially lessens the need for management actions to reduce the size of winter migration into Paradise Valley. Hunting, however, remains a vital interest among the local community. Our model reveals that by shifting the focus of the hunt away from cows, average hunt levels need not change dramatically in order to allow for a robust elk population and plenty of carrion for scavengers.

Our model builds upon the work of previous predator-prey and wolf-ungulate models (Crete et al., 1981; Hadjibiros, 1981; Stocker, 1981; Jensen and Miller, 2001; Miller et al., 2002) by incorporating a monthly time step so that seasonal carrion biomass to scavengers may be accounted for. We also expand upon the models of Miller et al. (2002) and Jensen and Miller (2001) by incorporating human hunters into our model. By explicitly keeping track of each year class of elk, we are able to tease out the differential effects of human hunters and wolves on elk population dynamics and carrion availability to scavengers. What emerges is a community perspective of predator-prey dynamics that so far has been ignored in these types of models.

Wolf reintroduction and re-colonization in other parts of the world may likewise affect scavenger species in those areas. Though species composition may change from location to location, the dynamics of carrion availability will likely respond in the same way. As such, conservation efforts focused on small and medium sized carnivores may benefit from 
the presence of wolves. In addition, management of wolves and/or human hunters should consider the synergism of these two predators when setting policy.

\section{Acknowledgements}

We thank Mary Power, Dale McCullough, Douglas Smith, Dan Stahler, James Lloyd-Smith, Paul Cross and Blake Suttle for their comments on this manuscript. The study was supported by an Environmental Protection Agency STAR fellowship to C. Wilmers.

\section{References}

Bangs, E.E., Fritts, S.H., 1996. Reintroducing the gray wolf to central Idaho and Yellowstone National Park. Wildl Soc. Bull. 24, 402-413.

Carbone, C., Du Toit, J.T., Gordon, I.J., 1997. Feeding success in African wild dogs: does kleptoparasitism by spotted hyenas influence hunting group size? J. Anim. Ecol. 66, 318326.

Crabtree, R.L., Sheldon, J.W., 1999. Coyotes and canid coexistence in Yellowstone. In: Clark, T.W., Curlee, A.P., Minta, S.C., Kareiva, P.M. (Eds.), Carnivores in Ecosystems: The Yellowstone Experience. Yale University Press, New Haven, pp. 429.

Crete, M., Taylor, R.J., Jordan, P.A., 1981. Simulating conditions for the regulation of a moose population by wolves. Ecol. Model. 12, 245-252.

Cross, P.C., Beissinger, S.R., 2001. Using logistic regression to analyze the sensitivity of PVA models: a comparison of methods based on African Wild Dog models. Conserv. Biol. 15, 13351346.

Estes, J.A., Palmisano, J.F., 1974. Sea otters: their role in structuring nearshore communities. Science 185, 1058-1060.

Farnes, P., Heydon, C., Hansen, K., 1999. Snowpack Distribution Across Yellowstone National Park. Final Report. Yellowstone Center for Resources No. 97-447.

Fuller, T.K., 1989. Population-dynamics of wolves in North-Central Minnesota. Wildl. Monogr. 1-41.

Gese, E.M., Ruff, R.L., Crabtree, R.L., 1996. Foraging ecology of coyotes (Canis latrans): the influence of extrinsic factors and a dominance hierarchy. Can. J. Zool. 74, 769-783.

Getz, W.M., 1996. A hypothesis regarding the abruptness of density dependence and the growth rate of populations. Ecology 77 , 2014-2026.

Hadjibiros, K., 1981. Simulation of a predator-prey system using a matrix model. Ecol. Model. 12, 45-67.

Haight, R.G., Mladenoff, D.J., Wydeven, A.P., 1998. Modeling disjunct gray wolf populations in semi-wild landscapes. Conserv. Biol. 12, 879-888.
Houston, D.B., 1978. Elk as winter-spring food for carnivores in northern yellowstone national park. J. Appl. Ecol. 15, 653-661.

Houston, D.B., 1982. The Northern Yellowstone Elk: Ecology and Management. Macmillan, New York.

Inouye, R.S., Byers, G.S., Brown, J.H., 1980. Effects of predation and competition on survivorship. Ecology 61, 1344-1351.

Jensen, A.L., Miller, D.H., 2001. Age structured matrix predation model for the dynamics of wolf and deer populations. Ecol. Model. 141, 299-305.

Krebs, J.R., Davies, N.B., 1981. An Introduction to Behavioral Ecology, 3rd ed. Blackwell Science Ltd, Oxford.

Lemke, T., Mack, J.A., Houston, D.B., 1998. Winter range expansion by the Northern Yellowstone elk herd. Intermount. J. Sci. 4, 1-9.

McLaren, B.E., Peterson, R.O., 1994. Wolves, moose, and tree rings on Isle Royale. Science 266, 1555-1558.

McNaughton, S.J., 1983. Serengeti grassland ecology: the role of composite environmental factors and contingency in community organization. Ecol. Monogr. 53, 291-320.

McNaughton, S.J., 1984. Grazing lawns-animals in herds, plant form, and coevolution. Am. Nat. 124, 863-886.

Mech, L.D., 1970. The Wolf: The Ecology and Behavior of an Endangered Species, 1st ed. American Museum of Natural History, Natural History Press, Garden City, NY.

Mech, L.D., Smith, D.W., Murphy, K.M., MacNulty, D.R., 2001. Winter severity and wolf predation on a formerly wolf-free elk herd. J. Wildl. Manage. 65, 998-1003.

Miller, D.H., Jensen, A.L., Hammill, J.H., 2002. Density dependent matrix model for gray wolf population projection. Ecol. Model. 151, 271-278.

Murphy, K.M., Felzien, G.S., Hornocker, M.G., Ruth, T.K., 1997. Encounter competition between bears and cougars: some ecological implications. Int. Conf. Bear Res. Manage. 10, 5560.

Newton, I., Davis, P.E., Davis, J.E., 1982. Ravens and buzzards in relation to sheep-farming and forestry in Wales. J. Appl. Ecol. 19, 681-706.

Paine, R.T., 1966. Food web complexity and species diversity. Am. Nat. 100, 65-75.

Paquet, P.C., 1992. Prey use strategies of sympatric wolves and coyotes in Riding Mountain National Park, Manitoba. J. Mammal. 73, 337-343.

Parker, K.L., Robbins, C.T., Hanley, T.A., 1984. Energy expenditures for locomotion by mule deer and elk. J. Wildl. Manage. 48, 474-488.

Peterson, R.O., Woolington, J.D., Bailey, T.N., 1984. Wolves of Kenai Penisula, Alaska. Wildl. Monogr. 88, 1-52.

Pitcairn, M.J., Getz, W.M., Williams, D.W., 1990. Resource availability and parasitoid abundance in the analysis of host-parasitoid data. Ecology 71, 2372-2374.

Power, M.E., 1990. Effects of fish in river food webs. Science 250, 811-814.

Ripple, W.J., Larsen, E.J., Renkin, R.A., Smith, D.W., 2001. Trophic cascades among wolves, elk and aspen on Yellowstone National Park's northern range. Biol. Conserv. 102, 227-234.

Singer, F.J., Harting, A., Symonds, K.K., Coughenour, M.B., 1997. Density dependence, compensation and environmental effects 
on elk calf mortality in Yellowstone National Park. J. Wildl. Manage. 61, 12-25.

Smith, D.W., Mech, L.D., Meagher, M., Clark, W.E., Jaffe, R., Phillips, M.K., Mack, J.A., 2000. Wolf-bison interactions in Yellowstone National Park. J. Mammal. 81, 1128-1135.

Stahler, D.R., Heinrich, B., Smith, D.W., 2002. Common ravens, Corvus corax, preferentially associate with gray wolves, Canis lupus, as a foraging strategy in winter. Anim. Behav. 64, 283290.

Starfield, A.M., Bleloch, A.L., 1986. Building Models for Conservation and Wildlife Management. Burgess International Group INC, Edina, MN, USA.

Stocker, M., 1981. Optimization model for a wolf-ungulate system. Ecol. Model. 12, 151-172.

Taper, M.L., Gogan, P.J.P., 2002. The Northern Yellowstone elk: density dependence and climatic conditions. J. Wildl. Manage. $66,106-122$
Vucetich, J.A., Peterson, R.O., Schaefer, C.L., 2002. The effect of prey and predator densities on wolf predation. Ecology 83, 3003-3013.

Wilmers, C.C., Crabtree, R.L., Smith, D., Murphy, K.M., Getz, W.M., 2003a. Trophic facilitation by introduced top predators: gray wolf subsidies to scavengers in Yellowstone National Park. J. Anim. Ecol. 72, 909-916.

Wilmers, C.C., Stahler, D.R., Crabtree, R.L., Smith, D.W., Getz, W.M., 2003b. Resource dispersion and consumer dominance: scavenging at wolf- and hunter-killed carcasses in Greater Yellowstone, USA. Ecol. Lett. 6, 996-1003.

Wisdom, M.J., Mills, L.S., 1997. Sensitivity analysis to guide population recovery: prairie-chickens as an example. J. Wildl. Manage. 61, 302-312.

Wisdom, M.J., Mills, L.S., Doak, D.F., 2000. Life-stage simulation analysis: estimating vital-rate effects on population growth for conservation. Ecology 81, 628-641. 